\title{
Clinical, epidemiological, and microbiological characteristics of bacteremia caused by high-level gentamicin-resistant Enterococcus faecalis
}

\author{
A.G. Vigani, A. Macedo de Oliveira, O.J. Bratfich, R.S.B. Stucchi and M.L. Moretti \\ Disciplina de Doenças Infecciosas e Parasitárias, Departamento de Clínica Médica, Faculdade de \\ Ciências Médicas, Universidade Estadual de Campinas, Campinas, SP, Brasil
}

Correspondence to: M.L. Moretti, Departamento de Clínica Médica, Hospital de Clínicas, Unicamp, Caixa Postal 6198, 13083-970 Campinas, SP, Brasil

Fax +55-19-3289-4107. E-mail: moretti@hc.unicamp.br

\begin{abstract}
Enterococcus spp bacteremia is associated with high mortality and the appearance of high-level gentamicin resistance (HLGR) created additional challenges for the treatment of these infections. We evaluated the epidemiological and clinical characteristics of patients with bacteremias caused by HLGR and non-HLGR Enterococcus faecalis isolates at a teaching hospital in the State of São Paulo, Brazil. Patients with bacteremia due to E. faecalis diagnosed between January 1999 and December 2003 were included in the study. We collected clinical, epidemiological, and microbiological data from medical records. Banked isolates were typed using pulsed-field gel electrophoresis. We identified 145 cases of $E$. faecalis bacteremia: 66 (45.5\%) were caused by HLGR isolates and 79 (54.5\%) by non-HLGR. In the univariate analysis, patients with HLGR infection were older, had higher rates of bladder catheterization, and more often had treatment with cephalosporin, quinolone, and/or carbapenem compared with patients with non-HLGR infection $(P<0.05)$. Multivariate analysis indicated that older age, hematological malignancy, and previous use of vancomycin were independently associated with HLGR $(P<0.05)$. Mortality rates were not significantly different among patients with HLGR $(50 \%)$ and non-HLGR $(43 \%)$ infections $(P=0.40)$. Of the 32 genotyped isolates, 16 were distributed into 6 main electrophoresis patterns and 16 others had distinct patterns. E. faecalis bacteremia is associated with high mortality and is frequently caused by HLGR isolates at this teaching hospital. The variability among genotyped isolates suggests that endogenous infections, rather than patient-to-patient transmission of $E$. faecalis, are more common at this institution.
\end{abstract}

Key words: Enterococcus faecalis; Bacteremia; High-level gentamicin resistance; Infection control; Enterococcus faecalis genotyping

Publication supported by FAPESP.

Received November 8, 2007. Accepted October 17, 2008

\section{Introduction}

Enterococcus spp is the third most frequent cause of hospital-acquired bacteremia, accounting for $8 \%$ of all nosocomial bacteremias in the United States (1). Highlevel gentamicin resistance (HLGR) has emerged among Enterococcus spp over the last two decades, and is associated with the loss of synergy between cell-active antibiotics (e.g., vancomycin and penicillin, particularly ampicillin) and most aminoglycosides (e.g., gentamicin, tobramycin, amikacin, and kanamycin), thus posing additional treatment challenges (2).

Enterococcal bacteremia is associated with high mortality, which is even further increased when caused by HLGR strains compared with those caused by non-HLGR strains (3-5). In Brazil, clinical and epidemiological studies of enterococcal infections have been less comprehensive, with most studies focusing on vancomycin-resistant En- 
terococcus (VRE), which is still restricted to a few Brazilian hospitals (6-8).

Since Enterococcus spp is the second most common cause of Gram-positive blood stream infections in Brazil and that approximately $76 \%$ of all enterococcal infections are due to Enterococcus faecalis, we evaluated cases of bacteremia caused by this organism at the teaching hospital of the Universidade Estadual de Campinas (Unicamp), Campinas, São Paulo State (8). Our objective was to characterize the epidemiological, clinical, and microbiological features of bacteremias caused by HLGR and nonHLGR E. faecalis isolated from January 1999 through December 2003. This evaluation could help identify measures to reduce the frequency of these infections, especially when caused by HLGR bacteria.

\section{Patients and Methods}

\section{Hospital setting}

The teaching hospital of Unicamp is a 403-bed, tertiary-care hospital that serves an estimated population of 5 million people. During the study period, there were approximately 14,000 admissions per year.

\section{Study population}

Patients with $E$. faecalis bacteremia were identified through a review of blood-culture records at the hospital microbiology laboratory. In accordance with current guidelines, E. faecalis bacteremia was defined as at least one positive blood culture for this pathogen (9). In patients with multiple episodes of bacteremia, only the first episode was considered for inclusion in this study.

\section{Study design}

We reviewed patient medical records using standard forms and collected data on potential risk factors for enterococcal infections. These included epidemiological and clinical information (age, gender, hospitalization ward, primary diagnosis, underlying chronic diseases, and severity of disease) and microbiological data (monomicrobial or polymicrobial etiology and antimicrobial susceptibility of $E$. faecalis). The severity of disease was estimated using the Charlson score (10). Invasive procedures prior to bacteremia onset, such as surgery, urinary bladder catheterization, mechanical ventilation, central venous catheterization, and administration of antimicrobials prior to the identification of $E$. faecalis, were also recorded.

\section{Definitions}

Community-acquired $E$. faecalis bacteremia was defined as a positive blood culture drawn $<72 \mathrm{~h}$ from patient admission or evidence of infection at the time of admission. Nosocomial E. faecalis bacteremia, conversely, was defined as a positive blood culture drawn $\geq 72 \mathrm{~h}$ after admission and absence of any evidence of infection at admission.

Identification and antimicrobial susceptibility testing

Enterococcus species and antimicrobial susceptibility were determined using the Gram-Positive Identification Card $^{\circledR}$ and Gram-Positive Susceptibility Card ${ }^{\circledR}$, respectively (bioMérieux Vitek ${ }^{\circledR}$, Marcy l'Étoile, France). A minimum inhibitory concentration of $500 \mu \mathrm{g} / \mathrm{mL}$ was used as the cutoff value for HLGR according to manufacturer guidelines.

\section{Genotyping}

DNA was extracted as described previously (11). Purified DNA was digested with the restriction enzyme Smal and products were separated by pulsed-field gel electrophoresis (PFGE) on 1.0\% agarose gels using CHEF-DRIII (Bio-Rad Laboratories, USA). Pulse time was increased from 5 to $35 \mathrm{~s}$, at $6.0 \mathrm{~V} / \mathrm{cm}$ for $20 \mathrm{~h}$ at $14^{\circ} \mathrm{C}$. Gel images were captured and analyzed using autoradiographic images from IS-1000 digital imaging system (Bio-Capt MW, version 99, M\&S Instruments Trading Inc., France). We estimated the relationship among the isolates using the Dice coefficient (Biogene software, Vilbert-Loumart, France). Isolates with similarity $<85 \%$ by PFGE were considered to be genetically unrelated, while similarity of $\geq 85 \%$ was considered evidence of isolates belonging to the same PFGE subtype (12). PFGE profiles were identified by capital letters.

\section{Statistical analysis}

Univariate analysis was performed using logistic regression, and the Fisher exact and Mann-Whitney tests, when appropriate. Variables for which an association with HLGR was suspected (i.e., P value $\leq 0.20$ ) were included in a multivariate stepwise logistic regression model. We considered the $P$ value $<0.05$ to be statistically significant.

\section{Results}

A total of 145 cases of bacteremia caused by $E$. faecalis were identified: 66 (45.5\%) were HLGR and 79 (54.5\%) non-HLGR. Cases of nosocomial bacteremia numbered 115 (80\%). Polymicrobial bacteremia was present in 70 $(48.3 \%)$ cases and Gram-negative bacilli were isolated in $42(60 \%)$ of those.

Gender distribution was similar in patients with HLGR and non-HLGR infections (Table 1). In addition, patients with HLGR were older than patients without HLGR, median 
age of 48.5 years (range: $10-81$ years) versus 38 years (range: 1 day-80 years), respectively $(P=0.008)$. Length of hospitalization prior to bacteremia diagnosis did not differ significantly between patients with HLGR (14 days) and non-HLGR (13 days) infection $(P=0.47)$. Among underlying chronic diseases, only hematological malignancies were significantly associated with HLGR bacteremia $(P=$ 0.005; Table 1).

The association between any antimicrobial therapy and the presence of HLGR bacteremia approached statistical significance $(P=0.06)$. However, when each antimicrobial was evaluated individually, HLGR was associated with previous use of quinolone [odds ratio $(\mathrm{OR})=2.79$, $95 \%$ confidence interval $(\mathrm{Cl})=1.19-6.53$ ], carbapenem $[\mathrm{OR}=4.52,95 \% \mathrm{Cl}=1.19-17.20]$, and cephalosporin [OR
$=1.98,95 \% \mathrm{Cl}=1.02-3.84 ;$ Table 1$]$.

Urinary catheterization was found to be associated significantly with HLGR bacteremia $(P=0.02)$. We were unable to show an association of other invasive procedures and HLGR bacteremia (Table 1).

Multivariate stepwise logistic regression analysis showed that age (at 10-year intervals; adjusted OR $=1.33$, $95 \% \mathrm{Cl}=1.13-1.57$ ), hematological malignancies (adjusted $\mathrm{OR}=4.92,95 \% \mathrm{Cl}=1.82-13.27)$, and previous use of vancomycin (adjusted OR $=3.93,95 \% \mathrm{Cl}=1.06-14.52$ ) were each associated independently with HLGR infection.

Of the 145 patients, 67 (46.2\%) died during hospitalization. Bacteremia by HLGR isolates did not influence patient outcome; 33 (50\%) of 66 patients with HLGR bacteremia died versus 34 (43\%) of 79 with non-HLGR bacter-

Table 1. Univariate analysis of factors associated with high-level gentamicin-resistant (HLGR) and non-HLGR Enterococcus faecalis bacteremia.

\begin{tabular}{|c|c|c|c|c|}
\hline Variables & HLGR $(N=66)$ & Non-HLGR $(\mathrm{N}=79)$ & OR & $95 \% \mathrm{Cl}$ \\
\hline Gender (male) & $34(51.5 \%)$ & $44(55.7 \%)$ & 1.18 & $0.61-2.28$ \\
\hline \multicolumn{5}{|l|}{ Age } \\
\hline $0-14$ years & $1(1.5 \%)$ & $24(30.4 \%)$ & & \\
\hline $14-64$ years & $49(74.3 \%)$ & $40(50.6 \%)$ & & \\
\hline$>65$ & $16(24.2 \%)$ & $15(19.0 \%)$ & & \\
\hline For each 10 years & & & 1.27 & $1.09-1.47$ \\
\hline Charlson score, median (range) & $2(0-6)$ & $2(0-9)$ & & \\
\hline At least 1 underlying diagnosis ${ }^{a}$ & $61(92.4 \%)$ & $67(84.8 \%)$ & 2.19 & $0.73-6.56$ \\
\hline Hypertension & $14(21.2 \%)$ & $14(17.7 \%)$ & 1.25 & $0.55-2.85$ \\
\hline Solid malignancy & $11(16.7 \%)$ & $14(17.7 \%)$ & 0.93 & $0.39-2.21$ \\
\hline Hematological malignancy & $18(27.8 \%)^{*}$ & 7 (8.9\%) & 3.86 & $1.50-9.94$ \\
\hline Chronic renal failure & $11(16.7 \%)$ & $10(12.6 \%)$ & 1.38 & $0.55-3.49$ \\
\hline Diabetes mellitus & $10(15.0 \%)$ & $8(10.1 \%)$ & 1.59 & $0.59-4.28$ \\
\hline \multicolumn{5}{|l|}{ Prior use of antimicrobials } \\
\hline Any antimicrobial & $46(70.0 \%)$ & $43(54.4 \%)$ & 1.93 & $0.97-3.83$ \\
\hline Aminoglycoside & 7 (10.61\%) & $14(17.7 \%)$ & 0.55 & $0.21-1.46$ \\
\hline Carbapenem & $10(15.1 \%)^{*}$ & $3(3.8 \%)$ & 4.52 & $1.19-17.2$ \\
\hline Cephalosporin & $37(56.0 \%)^{*}$ & $31(39.2 \%)$ & 1.98 & $1.02-3.84$ \\
\hline Metronidazol & 19 (28.8\%) & $14(17.7 \%)$ & 1.88 & $0.86-4.12$ \\
\hline Penicillins & $5(7.60 \%)$ & $13(16.5 \%)$ & 0.42 & $0.14-1.24$ \\
\hline Quinolone & $19(28.8 \%)^{*}$ & $10(12.6 \%)$ & 2.79 & $1.19-6.53$ \\
\hline Vancomycin & $9(13.6 \%)$ & $4(5.0 \%)$ & 2.96 & $0.87-10.1$ \\
\hline Others & $1(1.5 \%)$ & $5(6.3 \%)$ & 0.23 & $0.03-2.00$ \\
\hline \multicolumn{5}{|l|}{ Hospital procedures } \\
\hline Central venous catheter & $46(69.7 \%)$ & $51(64.6 \%)$ & 1.26 & $0.63-2.54$ \\
\hline Urinary catheter & $44(66.7 \%)^{\star}$ & $38(48.1 \%)$ & 2.16 & $1.10-4.24$ \\
\hline Mechanical ventilation & $22(33.3 \%)$ & $27(34.2 \%)$ & 0.96 & $0.48-1.92$ \\
\hline Surgery & $22(33.3 \%)$ & $25(31.6 \%)$ & 1.08 & $0.54-2.17$ \\
\hline Polymicrobial bacteremia & $36(54.5 \%)$ & $34(43.0 \%)$ & 1.59 & $0.82-3.07$ \\
\hline
\end{tabular}

Data are reported as number with percent in parentheses. aSome patients had more than one underlying diagnosis. ${ }^{*} \mathrm{P}<0.005$ compared to non-HLGR bacteria (univariate logistic regression). 
emia $(P=0.40)$.

Evaluation of antimicrobial resistance revealed that 40 $(27.6 \%)$ E. faecalis isolates had high-level resistance to streptomycin alone, 84 (57.9\%) had high-level resistance to either gentamicin or streptomycin, and 22 (33.3\%) had high-level resistance to both gentamicin and streptomycin. Resistance to ampicillin was present in $3 \%$ of isolates and was not significantly associated with HLGR ( $P=0.60)$. Additionally, the prevalence of resistance to ciprofloxacin was significantly higher among HLGR than non-HLGR isolates (92.4 vs $11.4 \%$, respectively; $P<0.001)$. Vancomycin resistance was not detected in any isolate.

Only $32(22 \%)$ E. faecalis isolates were available for PFGE. We constructed a dendrogram based on PFGE results (Figure 1). Sixteen isolates were grouped in six main PFGE patterns. The remaining 16 isolates had distinct PFGE profiles. Molecular profiles $D$ and $F$ included HLGR $E$. faecalis isolates only, while all isolates in group $E$ were non-HLGR. PFGE profiles $A, B$, and $C$ were comprised of HLGR and non-HLGR $E$. faecalis isolates. Of the six PFGE profiles with more than one isolate, profiles $A, C$, $D$, and $F$ had isolates collected from patients admitted to hospital wards $\geq 30$ feet apart.

\section{Discussion}

Enterococcus spp has gained importance over the last years partially due to its increase in prevalence and the emergence of multiresistant strains in the hospital setting. The prevalence of HLGR enterococcal bacteremia has been found to be between 7 and 63\% in North American hospitals $(5,13,14)$. This wide variation might be explained by differences in study designs, sources of specimens, and species considered. Previous studies of antimicrobial susceptibility of Enterococcus spp in Brazil, not focused on $E$. faecalis and with samples from different sites (blood, urine, and sputum), found the prevalence of HLGR to range from 27 to $55 \%(6,7)$. In our study, the first to focus on $E$. faecalis bacteremia in Brazil, $45 \%$ of cases were due to HLGR isolates.

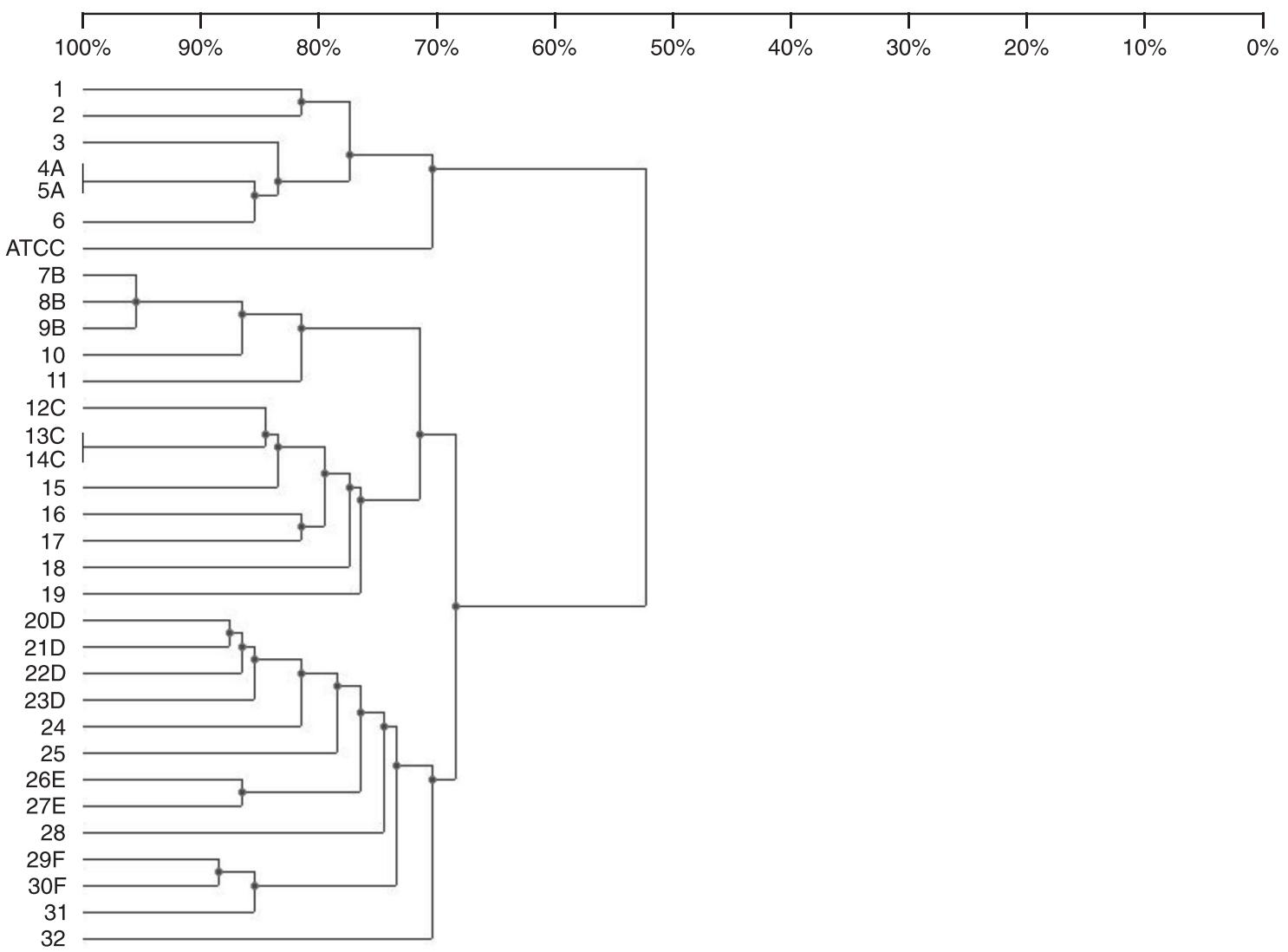

Figure 1. Dendrogram of pulsed-field gel electrophoresis profiles of 32 Enterococcus faecalis isolates (numbered isolates) and American type culture collection (ATCC) E. faecalis reference strain. 
The ability of Enterococcus to survive in the environment facilitates the survival of multidrug-resistant strains, creating more opportunities for person-to-person transmission to occur. This attribute suggests that Enterococcus and its resistance to antimicrobial drugs could continue to pose a challenge for both clinicians and hospital epidemiologists. In addition, the concomitant isolation of enterococci and other microorganisms in blood cultures has been reported in $24-49 \%$ of cases $(3,4,14,15)$. This can make therapeutic decisions even more difficult and potentially worsen prognosis. We observed a polymicrobial bacteremia prevalence of $48 \%$ with no significant difference between HLGR and non-HLGR isolates. However, the association of gentamicin-resistant strains and polymicrobial infections has not been conclusively established in the literature (13-15).

Patients with HLGR infections were significantly older than patients with non-HLGR infections. Previous studies, however, have not shown this difference comparing HLGR and non-HLGR E. faecalis infections $(5,13,16)$. A low prevalence of HLGR, $4 \%$, has been reported in a pediatric hospital (17). We speculate that the use of antimicrobials could be more judicious in pediatric patients, creating less selective pressure on drug-resistant bacteria. Additionally, the SENTRY Antimicrobial Surveillance Program has identified age-associated differences in pathogen prevalence and antimicrobial susceptibility among organisms causing bloodstream infections in North American hospitals (18). This program found, for example, that bloodstream infections caused by VRE occurred more frequently in adults than pediatric patients. It is unclear why age-related differences in antimicrobial susceptibilities occur.

In our study, hematological malignancies were the only underlying disease or condition significantly associated with higher rates of bacteremias caused by HLGR. Other studies have shown association of HLGR infection with chronic renal failure (16). We hypothesize that our results may be related to the practice at our hospital of using antimicrobial prophylaxis with quinolones among hematological patients, which might contribute to the selection of resistant strains. Similarly, an association between HLGR and resistance to ciprofloxacin among $E$. faecalis isolates has been described in the UK and Ireland (19). The use of glycopeptides, quinolones, carbapenems, and cephalosporins has also been associated with the emergence of gentamicin resistance and selection of HLGR strains in the past $(5,16)$. We found an association between HLGR and previous use of cephalosporin, quinolone, and carbapenen in the univariate analysis. This underscores the need for infection-control programs to promote judicious use of antimicrobials to decrease selective pressure on $E$. faecalis.
Our data showed that high-level resistance to both gentamicin and streptomycin was lower $(22 \%)$ than other studies $(41-82 \%)(5,14)$. Resistance to both of these aminoglycosides limits the treatment options in terms of combination therapy for severe enterococcal infections, such as endocarditis and meningitis. Of note, recommendations regarding the use of combination therapy for enterococcal bacteremia have not been delineated with certainty in the literature due to the absence of controlled trials to support it. However, infectious disease specialists recommend combination therapy for such infections, especially in critically ill patients (4).

The overall mortality among patients with enterococcal bacteremia was $46.2 \%$, which is consistent with previous reports $(30-68 \%)(3,4,15)$. Moreover, we found that mortality due to $E$. faecalis was similar regardless of the isolate's sensitivity to gentamicin, which is also consistent with the results of other studies $(5,13,14)$. The high mortality rates observed in patients with enterococcal bacteremia may reflect the severity of underlying illnesses. Unfortunately, we were not able to determine the contribution of $E$. faecalis infection alone on mortality because patients commonly had other risk factors that could be associated with death, e.g., polymicrobial infection and advanced age.

Molecular typing of Enterococcus isolates showed high variability among isolates, even among HLGR isolates. This molecular diversity suggests that isolates were selected from endogenous intestinal flora, rather than a result of patient-to-patient transmission. Similar findings have been reported by others (11). Nonetheless, we found a few $E$. faecalis isolates with similar PFGE patterns, suggesting that patient-to-patient transmission might have also occurred at our hospital. The finding of HLGR and non-HLGR isolates with the same PFGE pattern can be explained by the fact that PFGE is based on chromosomal DNA; HLGR phenotype, on the other hand, is associated with plasmids, which can be transferred independently from chromosomal DNA.

Our study is subject to several limitations. First, due to its retrospective design, it was not possible to use a more accurate index of severity of illness, such as the acute physiology and chronic health disease classification system II (APACHE II), and control for it in our analysis. Second, only a few of the isolates were available for genotyping, which limited our ability to evaluate transmission patterns. Finally, we focused on cases occurring over a four-year period, which may not be enough time for changes in antibiotic susceptibility to occur.

This study is the first to highlight the clinical, epidemiological, and microbiological characteristics of $E$. faecalis bacteremia at a large tertiary teaching hospital in Brazil. In 
addition, our results emphasize the need for strategies to improve infection-control practices, such as judicious use of antibiotics and monitoring of antibiotic resistance among hematologic patients. This could prevent further dissemi- nation of HLGR, allow the monitoring of changes in enterococcal resistance patterns, and ultimately prevent the spread of VRE at our institution, which is currently limited to a few cases of colonization (20).

\section{References}

1. Karlowsky JA, Jones ME, Draghi DC, Thornsberry C, Sahm DF, Volturo GA. Prevalence and antimicrobial susceptibilities of bacteria isolated from blood cultures of hospitalized patients in the United States in 2002. Ann Clin Microbiol Antimicrob 2004; 3: 7.

2. Moellering RC Jr. Emergence of Enterococcus as a significant pathogen. Clin Infect Dis 1992; 14: 1173-1176.

3. Malone DA, Wagner RA, Myers JP, Watanakunakorn C. Enterococcal bacteremia in two large community teaching hospitals. Am J Med 1986; 81: 601-606.

4. Maki DG, Agger WA. Enterococcal bacteremia: clinical features, the risk of endocarditis, and management. Medicine 1988; 67: 248-269.

5. Noskin GA, Till M, Patterson BK, Clarke JT, Warren JR. High-level gentamicin resistance in Enterococcus faecalis bacteremia. J Infect Dis 1991; 164: 1212-1215.

6. Cereda R, Pignatari AC, Hashimoto A, Sader H. In vitro antimicrobial activity against enterococci isolated in a university hospital in São Paulo, Brazil. Braz J Infect Dis 1997; 1: 83-90.

7. Sader HS, Sampaio JL, Zoccoli C, Jones RN. Results of the 1997 SENTRY Antimicrobial Surveillance Program in three Brazilian medical centers. Braz J Infect Dis 1999; 3: 63-79.

8. Titze-de-Almeida R, Rollo FM, Nogueira CA, Rodrigues IP, Eudes FJ, Nascimento RS, et al. Molecular epidemiology and antimicrobial susceptibility of Enterococci recovered from Brazilian intensive care units. Braz J Infect Dis 2004; 8: 197-205.

9. Garner JS, Jarvis WR, Emori TG, Horan TC, Hughes JM. CDC definitions for nosocomial infections, 1988. Am J Infect Control 1988; 16: 128-140.

10. Charlson ME, Pompei P, Ales KL, MacKenzie CR. A new method of classifying prognostic comorbidity in longitudinal studies: development and validation. J Chronic Dis 1987; 40: 373-383.

11. Murray BE, Singh KV, Heath JD, Sharma BR, Weinstock GM. Comparison of genomic DNAs of different enterococcal isolates using restriction endonucleases with infrequent recognition sites. J Clin Microbiol 1990; 28: 2059-2063.

12. Tenover FC, Arbeit RD, Goering RV, Mickelsen PA, Murray
BE, Persing DH, et al. Interpreting chromosomal DNA restriction patterns produced by pulsed-field gel electrophoresis: criteria for bacterial strain typing. J Clin Microbiol 1995; 33: 2233-2239.

13. Watanakunakorn C, Patel R. Comparison of patients with enterococcal bacteremia due to strains with and without high-level resistance to gentamicin. Clin Infect Dis 1993; 17: 74-78.

14. Antalek MD, Mylotte JM, Lesse AJ, Sellick JA Jr. Clinical and molecular epidemiology of Enterococcus faecalis bacteremia, with special reference to strains with high-level resistance to gentamicin. Clin Infect Dis 1995; 20: 103-109.

15. Gullberg RM, Homann SR, Phair JP. Enterococcal bacteremia: analysis of 75 episodes. Rev Infect Dis 1989; 11: 7485.

16. Caballero-Granado FJ, Cisneros JM, Luque R, Torres-Tortosa M, Gamboa F, Diez F, et al. Comparative study of bacteremias caused by Enterococcus spp. with and without high-level resistance to gentamicin. The Grupo Andaluz para el estudio de las Enfermedades Infecciosas. J Clin Microbiol 1998; 36: 520-525.

17. Christie C, Hammond J, Reising S, Evans-Patterson J. Clinical and molecular epidemiology of enterococcal bacteremia in a pediatric teaching hospital. J Pediatr 1994; 125: 392-399.

18. Diekema DJ, Pfaller MA, Jones RN. Age-related trends in pathogen frequency and antimicrobial susceptibility of bloodstream isolates in North America: SENTRY Antimicrobial Surveillance Program, 1997-2000. Int J Antimicrob Agents 2002; 20: 412-418.

19. Woodford N, Reynolds R, Turton J, Scott F, Sinclair A, Williams A, et al. Two widely disseminated strains of Enterococcus faecalis highly resistant to gentamicin and ciprofloxacin from bacteraemias in the UK and Ireland. $J$ Antimicrob Chemother 2003; 52: 711-714.

20. Tresoldi AT, Cardoso LG, Castilho GV, Dantas SR, von Nowakonski A, Pereira RM, et al. Low prevalence of vancomycin resistant enterococci colonization in intensive care patients in a Brazilian teaching hospital. Braz $J$ Infect Dis 2006; 10: 239-241. 